\title{
FULLSCALE TWO-CAR IMPACT TEST: A COMPARISON OF MEASURED AND MODEL RESULTS
}

\author{
Robert Rancatore \\ TIAX LLC \\ Cambridge, MA \\ Ronald Mayville \\ R.A. Mayville \& Associates \\ Newton, MA \\ Mark Baldwin \\ Zodiak Automotive U.S. \\ Phoenix, AZ \\ (Performed While at Simula, Inc.)
}

\begin{abstract}
The Volpe National Transportation Systems Center is conducting research into the crashworthiness of rail vehicles in support of the Federal Railroad Administration's Office of Research and Development. The approach taken has focused on the review of accidents, development of analytical tools and performing full-scale testing. A series of inline full-scale impact tests have been performed using conventional passenger cars. Recent full-scale testing included two instrumented coupled conventional passenger cars impacting a fixed barrier at $26 \mathrm{mph}$. The cars were instrumented with accelerometers, strain gages and string potentiometers. From these measurements, car translations, rotations, relative displacements and coupler forces were calculated. A rigid body dynamics model of the two-car configuration was developed and used to design the test. In order to improve the collision dynamics models of passenger cars, the results from this test are being used to refine that model.

This paper describes the two-car impact test, the reduction of data collected during the test and the refined collision dynamics model. Post-test refinements allow the model to more accurately simulate the vertical and lateral motions of the cars, including the timing of the lateral buckling of the cars. The post-test model also more accurately simulates the climbing of the impact car as it crushes. Comparisons between the refined model results with the measured data are presented for the motion of the center of gravity of the cars, coupled car interactions and forces, and lateral buckling.
\end{abstract}

\section{INTRODUCTION}

The Federal Railroad Administration's Office of Research and Development, with the assistance of the Volpe Center, has been conducting research studies on the crashworthiness behavior of conventional and modified passenger rail equipment. The goal of these studies is to enhance the safety of both passengers and crewmembers in the event of a derailment or collision. Work conducted for these studies includes the development of analytical models as well as scaled and fullscale testing. Examples of the models developed include: detailed three dimensional non-linear large deformation finite element crush models used to investigate the crush behavior of equipment and lumped parameter models to study the collision dynamic behavior of single cars, connected cars, and complete consists. The fidelity of the modeling approaches has been demonstrated by the close agreement of predicted responses with measurements taken from full-scale testing $[1,2,3]$ for key test parameters.

As part of this research, a series of tests are being carried out to compare the crashworthiness performance of conventional equipment and the performance of improved crashworthiness equipment [4]. The measurements made during these tests also allow the refinement of analytical models. As a result these refined models can predict the crashworthiness of trains with increased fidelity $[5,6]$.

A rigid barrier impact test was conducted on two coupled conventional Budd Company Pioneer rail passenger cars on 
April 4, 2000 [7]. The test was performed at the Transportation Technology Center (TTC) in Pueblo, Colorado. The principal objectives of the two-car test were to observe the failure modes of the major structural components, measure the gross motions of the cars, measure the force/crush characteristics of the impacting car and measure the interactions between the coupled cars.

Prior to the test, collision dynamics analyses were performed to design the test. The test was designed so that there was intrusion into the space for the first row of passenger seats in the impact car and lateral buckling at the coupled interface. The model predictions were used to determine the test speed, and to specify the test instrumentation.

\section{TEST DESCRIPTION}

On April 4, 2000, an impact test was conducted of two conventional cars traveling at approximately $26 \mathrm{mph}$ and impacting a rigid barrier. Figure 1 is a schematic of the test.

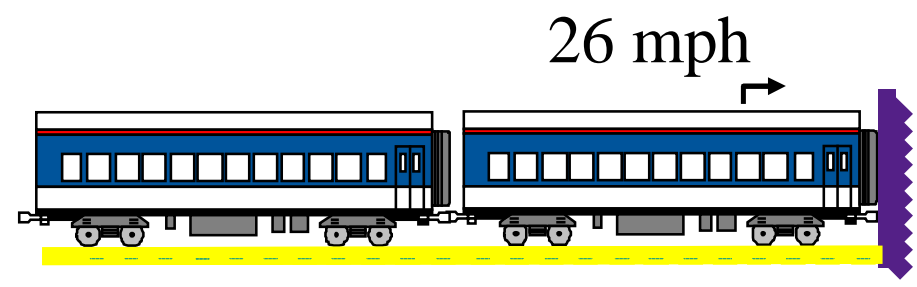

Figure 1: Schematic of Two-Car Full-Scale Impact Test

The original seats and ancillary equipment had been removed from both cars. Approximately 10,000 pounds of concrete ballast was added to the floor of each car, bringing their weights to 71,000 and $77,000 \mathrm{lbs}$, for the impact and trailing cars, respectively. Instrumentation for the test included accelerometers, strain gages, displacement transducers and high-speed cameras [7].

The lead car was crushed, that is reduced in length, by approximately six feet during the test. There was little visible damage to the back end of the lead car or the leading end of the trailing car, although the ends of the cars appear to bump each other. During the initial loading of the lead car, the two cars showed relatively small lateral and vertical displacements.

The back end of the lead car and the leading end of the trailing car first bumped each other, and then the back end of the lead car shifted left and the lead end of the trailing car shifted right. This mode of coupled car interaction is referred to as sawtooth buckling. The front truck of the lead car displaced to the right allowing its wheels to rest on the concrete ties. The front right wheels of the trailing car derailed as the left-side track rolled outwards during the impact event. The rails bent laterally into an "S" shape but remained parallel, the rails and ties moving together. As the cars came to rest, the lead end of the trailing car was pushed to the left and the rear end of the leading car was pushed to the right.

The impact end of the lead car can be seen rising by approximately 9 inches as the carbody structure crushes. As the lead car crushed, the front end rose up against the barrier due to local deformation of the draft sill, causing the rear end of the car to lower. As the trailing car continued moving forward, it attempted to override the lead car. The trapping of the coupler below the underframe of the vehicles apparently prevented further upward motion.

\section{DATA REDUCTION AND MODELING APPROACH}

The approach taken in this study was to first process the test data, and then refine the model. The steps taken to process the test data include:

1. The film and video were processed to determine the velocities and displacements of the vehicles.

2. The accelerometer data were filter and integrated to determine the velocities and displacements of the vehicles and compared to the film and video data, where applicable.

3. The strain data was processed to determine forces on the coupler.

4. The string potentiometer data were analyzed to determine displacements of the coupler.

Using the processed data, the steps taken to refine the model and verify these refinements include:

1. The input parameters for the model, including the carbody masses, moments of inertia, location of the center of gravity, etc., were changed as necessary, based on the as-tested conditions of the cars.

2. Comparing the pre-test model predictions, using the verified input parameters, with the processed test data, helped identify the features of the model to be changed.

3. The model changes identified in Step 2 were implemented

4. The model changes were verified by comparing the refined model predictions with the processed test data.

Steps 2, 3, and 4 were repeated until the model results were in close agreement with the test measurements.

This approach allows identification of the model features necessary for a high level of fidelity in simulating train collisions. Comparison of the two-car test measurements with pre-test model results showed close agreement for the crush and deceleration of the lead car, and close agreement for the deceleration of the trailing car [7]. The model results for the lateral and vertical motions of the cars, and the interactions of the coupled cars are qualitatively consistent with the test data, but do not agree closely quantitatively. This approach allowed identification of the model features necessary to simulate the vertical and lateral interaction of coupled cars accurately.

The measurements made during the test and their processing are described in the Appendix. The refined model, including a comparison of selected model results and corresponding test measurements, are presented in the body of the paper.

\section{MODEL DESCRIPTION}

A rigid body collision dynamics model, implemented in ADAMS [8], was developed to help design the two-car test [7]. Figure 2 shows a schematic of the pre-test model. This model uses discrete masses connected by non-linear springs and dampers. The lead car in the model consists of four rigid masses. Two of the four masses represent carbody mass while the remaining two masses are represented by the trucks. At the front of the vehicle is a mass that represents the crushing structure of the vehicle. It is connected to the remaining vehicle mass with several springs and dampers that represent the force/crush response of the center sill and side sills. The two truck masses are connected to the vehicle body by spring and dampers representing the secondary suspension of the vehicle. Forces applied at the pivot point between the vehicle body and 
the truck allow the truck to react to longitudinal and lateral forces on the vehicle and trucks. The trucks can pivot relative to the vehicle body. The trailing car is similar to the lead car, except that the carbody is represented by a single mass.

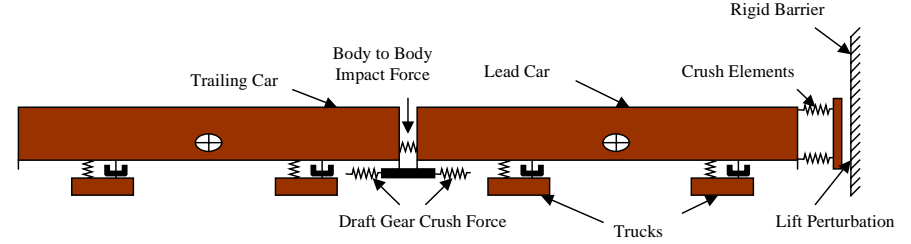

Figure 2. Two Car Collision Dynamics Model Schematic

The trailing car is connected to the lead car through a rigid link representing the couplers. The couplers are allowed to yaw and pitch at each end where they are connected to the vehicles. Non-linear springs and dampers connecting the couplers to the car bodies represent the draft gear and car body force/displacement responses.

Table 1 lists the masses and moments of inertia of the vehicles used in the post-test model. The weights of the cars did not change significantly from the pre-test to the post-test analysis, however, the roll, pitch, and yaw inertias did change. These values were revised based on the previous measurement of the roll, pitch, and yaw inertias of the car used in the single car test, as well as the locations for the ballast in the cars used in the two car test. These parameters are larger than the parameters used in the pre-test analysis, because most of the ballast was placed near the ends of the cars

Table 1. Model Mass and Moments of Inertia

\begin{tabular}{|c|c|c|c|c|}
\hline Vehicle & $\begin{array}{c}\text { Weight } \\
(\mathbf{l b m})\end{array}$ & $\begin{array}{c}\text { Roll } \\
\text { (lbm-ft }^{\mathbf{2}} \text { ) }\end{array}$ & $\begin{array}{c}\text { Pitch } \\
\left(\mathbf{l b m - f t}^{\mathbf{2}} \mathbf{)}\right.\end{array}$ & $\begin{array}{c}\text { Yaw } \\
\text { (lbm-ft }^{\mathbf{2}} \mathbf{)}\end{array}$ \\
\hline Lead Car & 71,000 & $1.78 \mathrm{e}+06$ & $3.73 \mathrm{e}+07$ & $3.73 \mathrm{e}+07$ \\
\hline Trail Car & 77,000 & $1.78 \mathrm{e}+06$ & $3.73 \mathrm{e}+07$ & $3.73 \mathrm{e}+07$ \\
\hline
\end{tabular}

The processed test measurements described in the Appendix were used to guide the refinements to the model. The model closely predicted the longitudinal motion of the cars during the test; however, it did not predict the lateral and vertical motions of the cars as well. Consequently, efforts were focused on the components of the model which most influence the predicted vertical and lateral car motions: the car-to-wall impact elements, the coupler elements between the cars, and the wheel/rail elements. Table 2 contains a list of the features added to the model to improve the model results and comparisons to the test data.

Several model features can potentially affect a particular result. For example, the vertical motion of the lead car is influenced by both the wall impact elements and by the coupler elements. The wheel/rail/ground interaction and the coupler elements affect the lateral buckling responses of both cars. These multi-feature dependencies make it difficult to improve the model results for the lateral and vertical motions of the cars without degrading the results for the longitudinal motions.
Table 2. Model Enhancements

- The lead car impact model was modified to allow lift of the impact end of the lead car.

- Separate draft gear and body crush elements replaced the single crush element of these responses in series.

- An elastic/plastic model of the draft gear was implemented to allow tension and compression in the draft gear.

- The friction-based wheel/rail model was refined to provide more control over the timing of the onset of lateral buckling.

As the vehicle impacted the wall, local deformation of the end of the vehicle caused the lead end of the impacting vehicle to lift, including the lead truck. The lift of the lead end lowered the trailing end of the impact car, creating a situation where the lead end of the trailing car could attempt to override the trailing end of the lead car. This interaction between the coupled cars affected the forces in the coupler as well as the eventual impact force between the vehicle bodies.

Heuristic elements are used to approximate the vertical motion of the car as it crushes. These elements are features added to the model, based on experience, to achieve a certain model response. The wall, rather than being perfectly flat, is represented by the surface of a 100-foot radius sphere. The vertical placement of the sphere allows for control of the rate of climb of the vehicle. The intent was to match the pitch rotation of the lead car in order to promote the interactions at the coupled interface. A second impact surface is used in the model to limit the climb of the vehicle. As the vehicle continues to climb and crush, it eventually dissipates enough energy to stop the climb and crush continues until the vehicle rebounds from the wall.

Modifications to the draft gear force/crush curve were also implemented. Figure 3 shows a comparison of the original and modified curves for force/crush of the draft gear element used in the model. This modification helped to change the magnitude of the vehicle-to-vehicle impact force and the timing of the force.

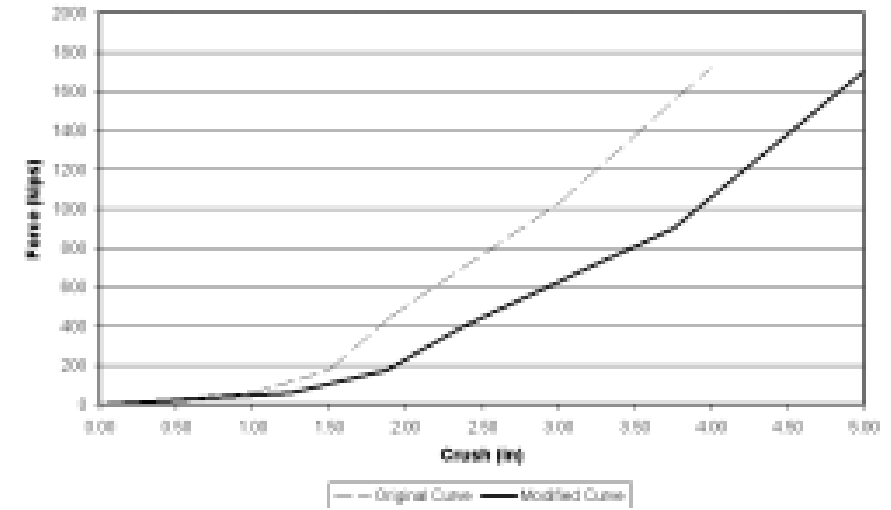

Figure 3: Original and Modified Draft Gear Force Crush Curves

Additionally, a one-way damping element was included in the draft gear model to provide the hysteresis response of the 
draft gear. The magnitude of the damping coefficient also affects the lateral buckling magnitude and timing due to its affect on the rebound response of the trailing car. A subroutine which allows tension and compression forces to be developed depending on the displacement condition was used to represent the draft gear. Figure 4 is a plot of the total draft gear force versus crush. The compression and expansion of the draft gear generates the hysteresis loops seen on the plot.

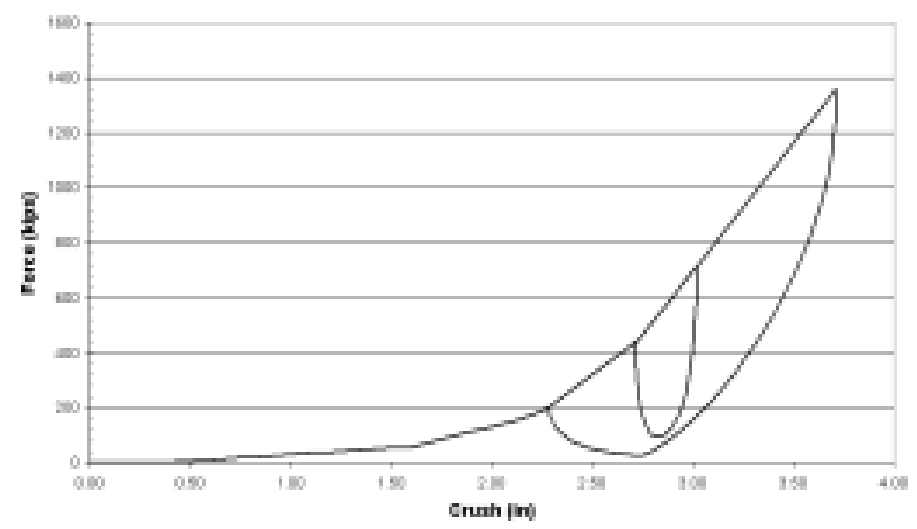

Figure 4: Simulated Lead Car Draft Gear Force vs Crush Response

The original truck/track representation used a single point of contact between the truck and each rail. This representation does not include sufficient detail to predict accurate timing of derailment, and consequently sawtooth buckling of the cars. Multiple wheel contact was implemented between the truck and each rail to accurately simulate the truck rotation. In addition, the wheel/rail friction-based representation was modified to include a lateral static friction component as a means of providing more control of the model in order to match the test results.

During normal operation, a lateral relative displacement of three to four inches may be seen at the coupled ends of vehicles due to suspension sway wheel/rail position. A wedge was placed in the suspension causing a one- to two-inch relative lateral displacement at the coupled end in an attempt to induce lateral bucking. During the test, the B-end of the lead car traveled laterally to the right, but remained on the track. The Aend of the trailing car traveled laterally to the left and also essentially remained on the track. The result is that the track deformed into an S-shape with the rails and cross-ties moving laterally together. The left rail also rolled in the vicinity of the lead truck of the trailing car with the result that the right-side wheels of the truck derailed causing the trailing car's right-front corner to come to rest at a lower elevation than the rear right corner.

Since the model does not explicitly include the rails, the friction model was able to approximate the force associated with the lateral displacement of the wheels. This representation uses several parameters to approximate the sequence of events during the sawtooth buckling of the cars. There are parameters for longitudinal friction on the rail (braking), static and dynamic parameters for lateral motion while a wheel set is on the rails, and there is a dynamic friction once the wheel has left the rail.

\section{COMPARISON OF MODEL AND TEST RESULTS}

Figures 5-10 compare the model's results to the measured data for performance parameters such as;

- longitudinal displacement of the CG,

- pitch angle of the lead vehicle,

- relative lateral displacement at the coupled ends,

- relative vertical displacement at the coupled ends,

- relative longitudinal displacement at the coupled end, and,

- the longitudinal coupler load.

Figure 5 shows the simulated and measured longitudinal displacement time-history of the lead car. The instant of impact is time zero. The simulated peak displacement is slightly larger than the peak measured displacement, but the timing of the peak is very close. The variation in the peak displacement is less than $5 \%$, well within the expected repeatability of the test.

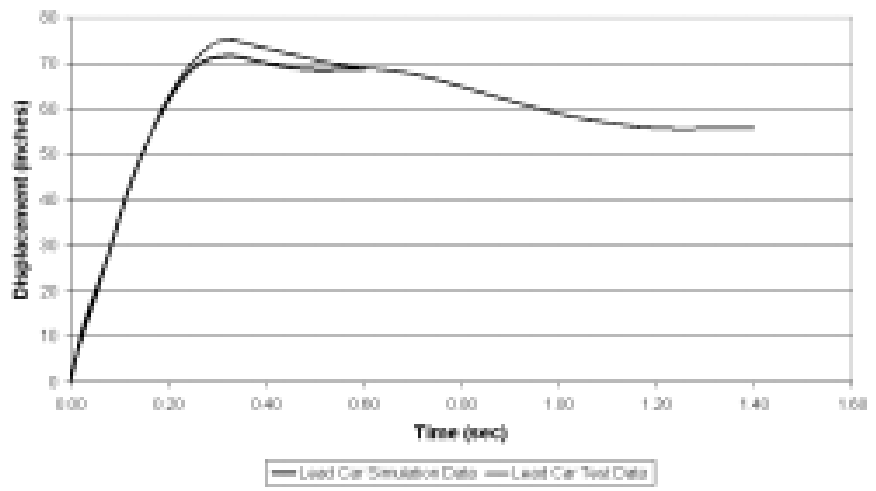

Figure 5: Simulated and Measured Longitudinal Displacement Time-History of the Lead Car Center of Gravity

Figure 6 contains a graph comparing the simulated lead car pitch rotation to the measured pitch rotation. The model simulates the rotation of the lead car well though 0.3 seconds. At that time the measured data levels off as the forward motion of the car stops (see Figure 5.) For the first 0.3 seconds, the model results and the test data are nearly identical. The model calculates a maximum displacement that is about $10 \%$ greater than the test measurement, and this peak occurs 0.05 seconds later in the model simulations than in the test measurements. 


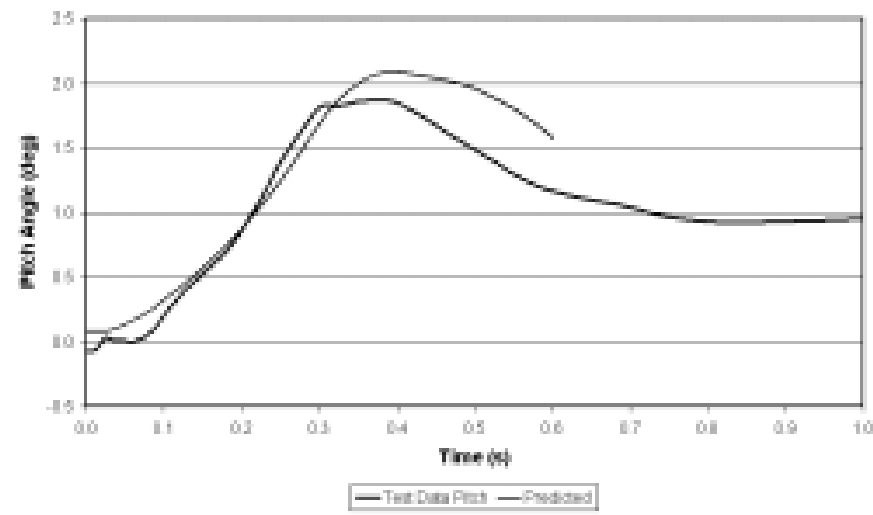

Figure 6: Simulated and Measured Lead Car Pitch Rotation versus Time

Figure 7 displays the simulated and measured relative lateral displacement of the coupled ends of the vehicles. This displacement is defined as the lateral distance between a point on the longitudinal centerline, at the end of the trailing car, relative to a point on the longitudinal centerline, at the end of the lead car. It is a measure of the sawtooth lateral buckling that occurred at the coupled interface. The model simulates lateral buckling at the same time as it occurred in the test. The model results for the peak lateral displacement is about $10 \%$ lower than the test data.

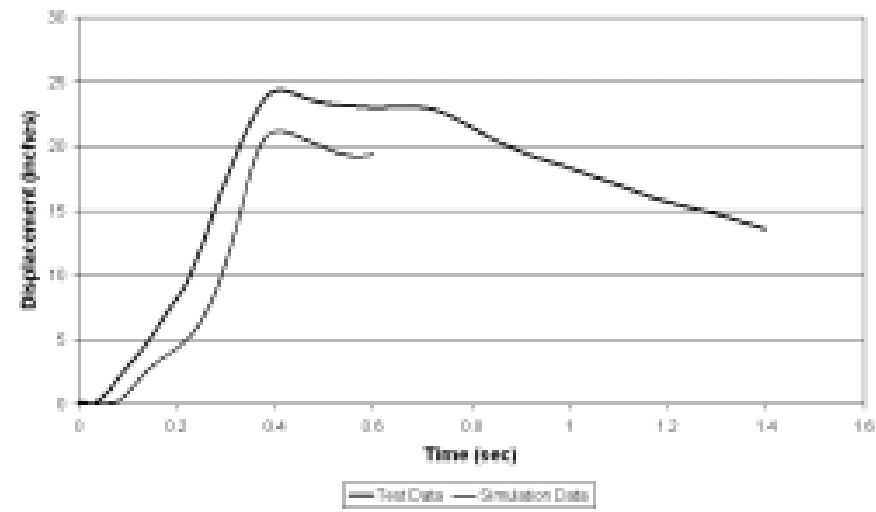

Figure 7: Simulated and Measured Relative Lateral Displacement Time-History at the Coupled End

Figure 8 shows the comparison of the simulated and measured relative vertical displacement time-histories at the coupled ends. This displacement is defined as the vertical distance between a point on the longitudinal centerline, at the end of the trailing car, relative to a point on the longitudinal centerline, at the end of the lead car. The measured data shows the A-end of the trailing car dropping below the B-end of the lead car immediately following the impact. This is due to an initial downward pitch of the lead car as it impacts the wall before the local end deformation provides the lift of the lead end. As the front of the lead car lifts, its trailing end drops. The orientation of the coupler allows a catapult-like motion of the A-end of the trailing car as the draft gear is being compressed. Eventually the coupler becomes trapped below the underframe and impacts the bellmouth stopping the override motion.

The refined model qualitatively captures the relative vertical displacement shown in Figure 8, but does not capture this motion quantitatively as closely as it captured the lateral motion. This variance is in part due to the heuristic car/wall element, which does not allow for the initial downward pitch of the lead car. The variance is also due in part to the coupler element. Potentially, the trailing end of the lead car was lower than the lead end of the trailing car immediately prior to the test, which allowed the catapult motion of the coupler to be more pronounced in the test.

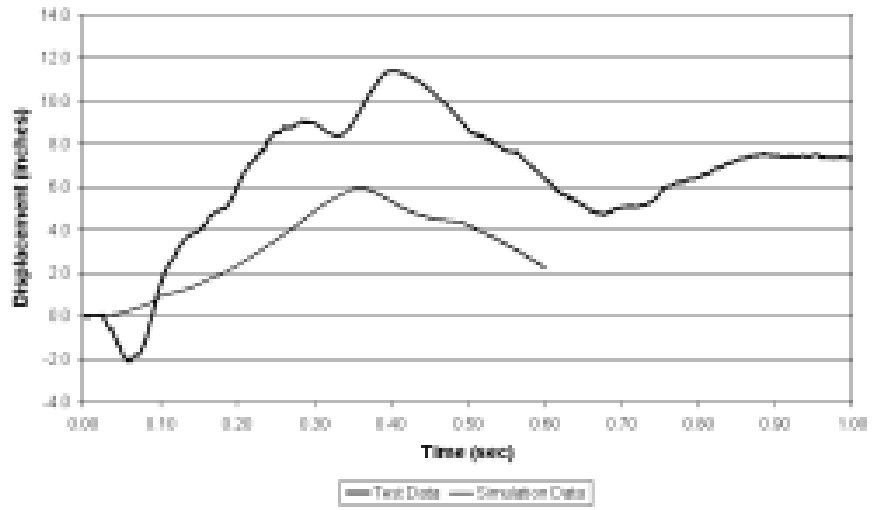

Figure 8: Simulated and Measured Relative Vertical Displacement Time-History at the Coupled Ends

Figure 9 contains the simulated and measured relative longitudinal displacement time-histories at the coupled ends. The simulated displacement tracks very well for almost 0.1 seconds. The form of the simulated curve qualitatively compares well with the test measurements. The measured data shows the trailing car end passing the lead car end near 0.25 seconds. This time is close to the time that the vehicle ends came in contact (see Figures A-6 and A-7 in Appendix). This indicates that the car bodies impacted while the trailing car attempted to override the lead car. Due to the method of implementing the impact forces between the car bodies, the simulated curve cannot simulate the penetration of the trailing car beyond the plane of the B-end of the lead car.

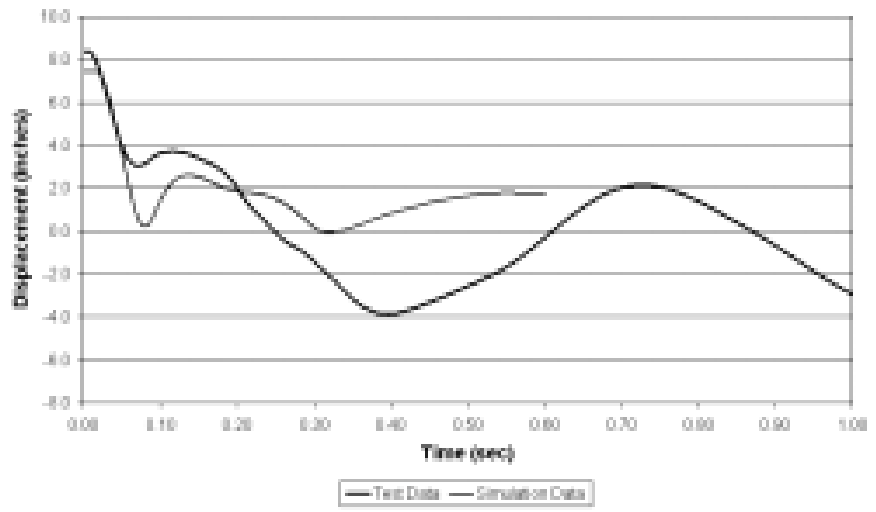

Figure 9: Simulated and Measured Relative Longitudinal Displacement Time-History at the Coupled Ends

Figure 10 shows the comparison of the measured and simulated longitudinal coupler loads. The magnitude and timing of the loads affect the response of the vehicle ahead of it as well as directly affecting the lateral buckling that occurs. The simulated timing and magnitude of the initial peak compares closely with the measured data. Although the local minimum of the simulated load is lower than that measured, the timing of it 
and the secondary peak is close to the timing of the measured peak.

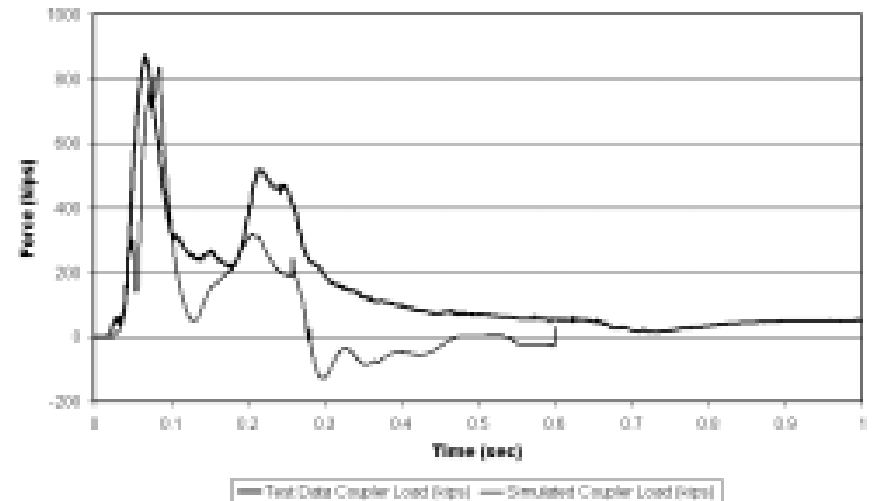

Figure 10: Simulated and Measured Longitudinal Coupler Load Time-History

\section{SUMMARY AND CONCLUSIONS}

The results of the two-car impact test have been used to develop a refined model of the test. These model refinements improved the model results for the lateral and vertical motions of the cars, without degrading the longitudinal results. The model can now simulate the lateral motions of both cars, including the relative lateral interaction and sawtooth buckling that occurred between the cars during the test. Using heuristic elements, the model can simulate the vertical motion of the lead car during the test. Additional study to improve the vertical response at the impact end of the vehicle will improve the model predictions. The amplitude of the vertical motion of the trailing car is lower than was measured in the test, although the model results and test measurements agree closely for the timing of minimum and maximum displacements. The variations in magnitude of these peaks may be due to differences in the initial vertical positions of the cars in the test and in the simulation.

The interactions of coupled passenger cars under impact conditions now are better understood. Key features of the coupler elements for accurately simulating the vertical and lateral motions of cars have been identified. Given the crush response of vehicles based on testing or crush analyses, the approach for modeling coupled passenger cars described in this paper can be used to develop models of longer trains, for simulating train response under a wide range of collision conditions.

\section{ACKNOWLEDGMENTS}

This work was performed under contract for the Volpe Center as part of the Equipment Safety Research Program sponsored by the Office of Research and Development of the Federal Railroad Administration. This contract was initiated and monitored by David Tyrell, Senior Engineer, Volpe Center. The authors would like to thank Dr. Tom Tsai, Program Manager, and Claire Orth, Division Chief, Equipment and Operating Practices Research Division, Office of Research and Development, Federal Railroad Administration, for their support. The authors would also like to thank Eloy Martinez, Karina Jacobsen and John Zolock of the Volpe Center and
Benjamin Perlman of Tufts University for their helpful review and suggestions.

\section{REFERENCES}

1. Tyrell, D., Severson, K., Perlman, A.B., Rancatore, R., "Train-to-Train Impact Test: Analysis of Structural Measurements," American Society of Mechanical Engineers, Paper No. IMECE2002-33247, November 2002.

2. Martinez, E., Tyrell, D., Zolock, J., "Rail-Car Impact Tests with Steel Coil: Car Crush," American Society of Mechanical Engineers, Paper No. JRC2003-1656, April 2003.

3. Jacobsen, K., Tyrell, D., Perlman, A.B. , "Rail-Car Impact Tests with Steel Coil: Car Crush," American Society of Mechanical Engineers, Paper No. JRC2003-1655, April 2003.

4. Tyrell, D., Tsai, N.T., "An Overview of Passenger Equipment Full-Scale Impact Tests," $4^{\text {th }}$ International Symposium on the Passive Safety of Rail Vehicles, October 7, 2003.

5. Kirkpatrick, S., MacNeil, R., "Development of a Computer Model for Prediction of Collision Response of a Railroad Passenger Car," Proceedings of the 2002 IEEE/ASME Joint Railroad Conference, Institute of Electrical and Electronics Engineers, Catalog Number CH37356-TBR, 2002.

6. Stringfellow, R. Rancatore, R., Llana, P. Mayville, R., "Analysis of Colliding Vehicle Interactions for the Passenger Train-to-Train Impact Test," American Society of Mechanical Engineers, Paper No. RTD2004-66037

7. Tyrell, D., K. Severson, J. Zolock, A.B. Perlman, "Passenger Rail Two-Car Impact Test Volume I: Overview and Selected Results," U.S. Department of Transportation, DOT/FRA/ORD-01/22.1, January 2002.

8. ADAMS, Version 12.0.0, Mechanical Dynamics, Inc. Ann Arbor, Michigan. 


\section{APPENDIX - TEST INSTRUMENTATION, DATA REDUCTION AND VEHICLE MOTIONS AND FORCES}

In order to provide a basis for comparison with the model results, the test data was reduced. Where necessary, adjustments were made to the reduced accelerometer data, based on the film data, to improve its consistency. A description of the adjustments is shown below. The overlapping nature of some of the measurements allowed these adjustments to be calculated. For example, the longitudinal displacement time histories of the cars can be determined by integrating accelerometer data and by motion analysis of high-speed film. Comparing both measurements allows the results of the test to be known with a high level of confidence.

\section{TEST INSTRUMENTATION}

Figure A-1 shows the mounting locations and type of accelerometer used for data collection on the leading and trailing cars. In Figure A-2, tri-axial accelerometers are shown as squares and uni-axial accelerometers as circles. Accelerometers were mounted at five longitudinal locations on the car, starting with Position No. 1 at the front of each car and ending with Position No. 5 at the rear of each car. On the lead car, tri-axial accelerometers were mounted along the center sill at Positions No. 2, No. 3, and No. 4, and along the left and right side rails at Position No. 3. Uni-axial accelerometers were mounted along the center, right and left side sills at Positions No. 1 and No. 5 and on the trucks at the front (B1) and rear (B2). On the trailing car, the tri-axial accelerometers were mounted in the same locations as on the leading car. In addition, three uni-axial accelerometers were mounted along the center sill at Position No. 1 and at the front and rear trucks.

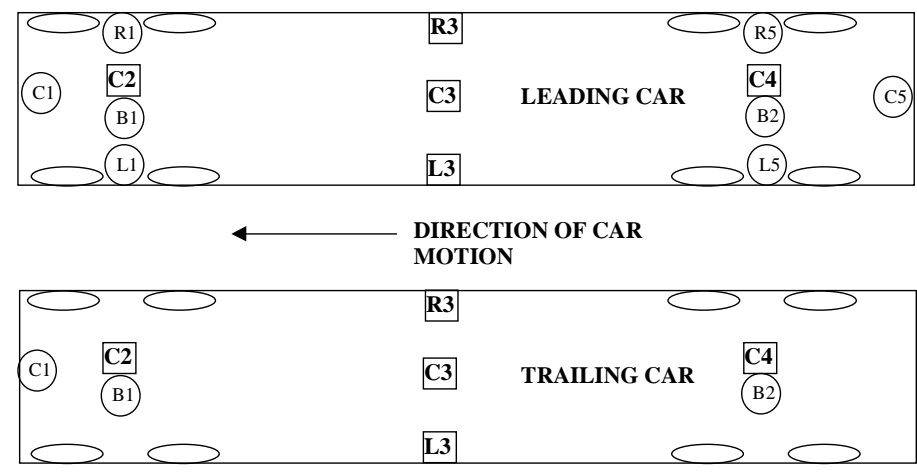

Figure A-1: Schematic of the Accelerometer Mounting Locations

Each coupler at the coupled interface was instrumented with three displacement transducers to measure its longitudinal, lateral and vertical displacements relative to its carbody. Figure A-2 shows a post-test photograph of the displacement transducers mounted between the coupler and the trailing car.

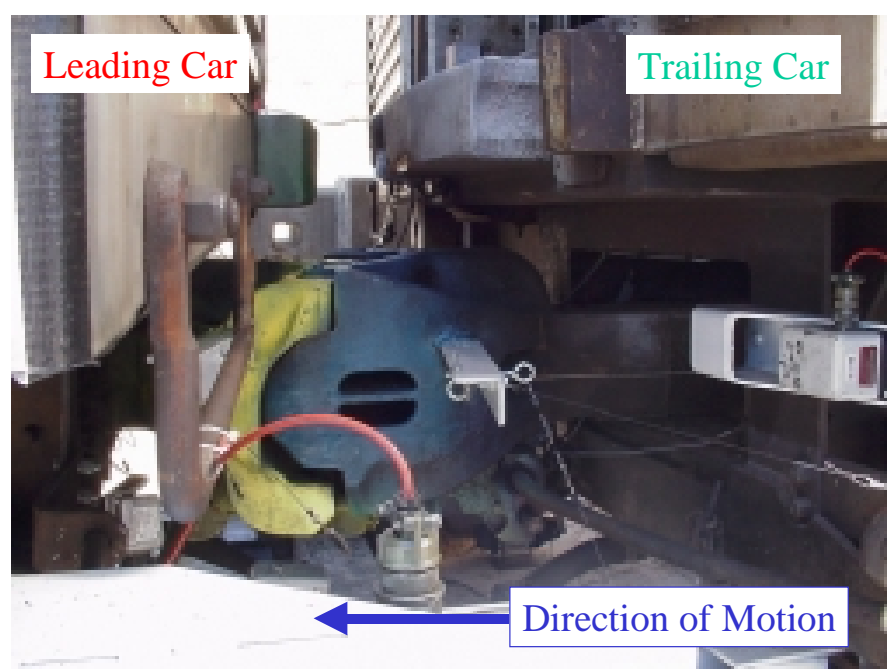

Figure A-2: Displacement Transducers Used to Measure Relative Displacements of the Coupler and the Trailing Car

High-speed cameras recorded the collision from several locations. Photometric analysis of the film was used to determine the displacements of the cars. The results of the photometric data were used to confirm the displacements calculated from the accelerometer data.

\section{DATA REDUCTION}

The film and sensor data were processed to determine gross car body center of gravity displacements and rotations, relative motion at the coupled interface, and coupler and body impact forces at the coupled interface. The film-based motion data was collected using two methods: 1) digital tracking of markers using converted computer video, and 2) hand tracking of markers on a grid to which the film of the test was projected. Due to limitations in tracking the markers (due to dust, impeding objects, or out-of-view camera framing), the digital method was not able to generate displacement data along all axes for both cars. However, displacements along all axes were generated using the hand tracking method. Table A-1 outlines the data that was collected for each car, the direction of the data, the camera views and locations, and which analysis method was used $(\mathrm{D}=$ digital$)$.

Table A-1. Summary of Motion Data Collected

\begin{tabular}{|l|l|l|}
\hline Motion Data Collected & Camera View & $\begin{array}{l}\text { Analysis } \\
\text { Type }\end{array}$ \\
\hline Leading X, Z, Front End & Side @ Barrier & D, Film \\
\hline Leading X, Y, Front End & Overhead @ Barrier & Film only \\
\hline Leading X, Z, Rear End & Side @ Coupler & D, Film \\
\hline Leading Y, Rear End & Overhead @ Coupler & Film only \\
\hline Trailing X, Z, Front End & Side @ Coupler & D, Film \\
\hline Trailing X, Y, Front End & Overhead @ Coupler & D, Film \\
\hline
\end{tabular}

Prior to conducting the post-test data analysis, a preliminary review of all accelerometer data was performed in an effort to eliminate poorly recorded data channels. Filtering for of the all channels processed was performed using a standard Class 180 SAE filter. All of the channels were timeshifted to account for $100 \mathrm{msec}$ of pre-trigger data collected in the data bricks prior to impact. 
An initial review of the raw and filtered accelerometer data revealed inconsistencies in some of the accelerometer data. As a result, the data collected from $\mathrm{C} 1 \mathrm{X}, \mathrm{L} 1 \mathrm{Z}$, and $\mathrm{R} 1 \mathrm{Z}$ were deemed unreliable, and were eliminated from further analysis. The irregularities in the data from $\mathrm{C} 2 \mathrm{X}$ are likely due to accelerometer failure. No inconsistencies were noted in the accelerometer profiles for the trailing car.

In order to determine the displacements based on the accelerometers, the data is double integrated. Errors in acceleration measurement can become magnified as a result of integration. Correction factors were used to improve the accuracy of the results. For example, the longitudinal velocity for the cars is zero at the completion of the test. Due to the accumulated errors, the calculated velocity at the end of the test from the accelerometer data for each of the leading car sensors was non-zero. To correct this, a baseline correction factor was calculated and added to each filtered accelerometer data set according to the following equation.

where:

$$
A_{\text {Corrected }}=A_{\text {Uncorrected }}+B C F
$$

$\mathrm{BCF}=$ Baseline Correction Factor, a constant acceleration offset value

Each baseline correction factor was less than 0.5 g's. A similar approach was used for the trailing car. Figure A-3 shows the uncorrected lead car longitudinal velocity vs. time plots for each sensor. Note the non-zero velocities at 1.4 seconds. Figure A-4 shows the corrected lead car longitudinal velocity vs time plots for each sensor. After applying the correction factors, the longitudinal velocities are essentially zero, signifying that the vehicle has come to rest after rebounding from the barrier wall.

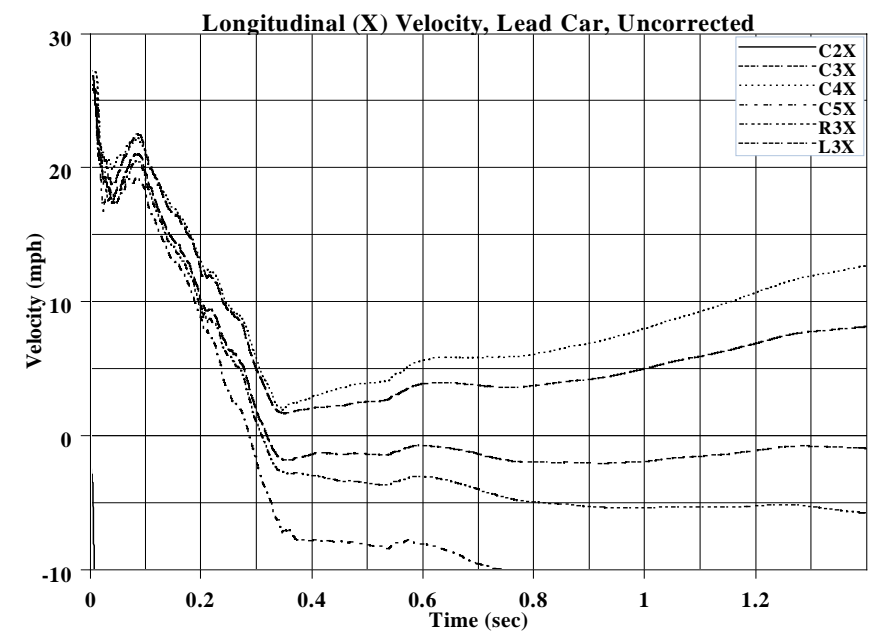

Figure A-3: Lead Car Longitudinal Velocity from Sensors Uncorrected

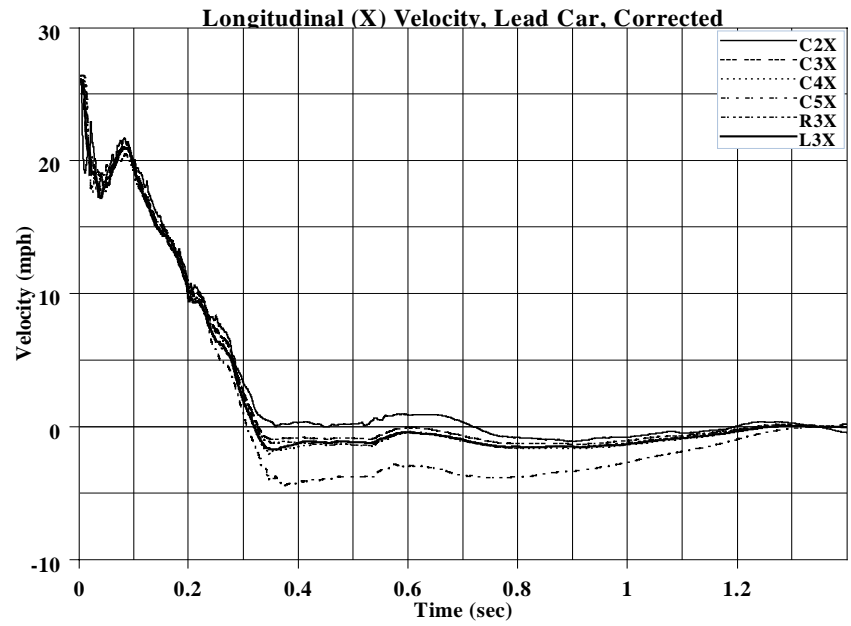

Figure A-4: Lead Car Longitudinal Velocity from Sensors Corrected

The lateral and vertical velocities calculated from the accelerometer data require three additional corrections: 1) a baseline correction factor to adjust the slope of the velocity; 2) a cross-axis contamination correction factor; and 3) a velocity offset correction factor. The correction factors applied to the velocity profiles are given by:

$$
V_{\text {Corrected }}=V_{\text {offset }}+(B C F \times t)+\left(C \times V_{\text {long,avg }}\right)
$$

where:

$$
\begin{aligned}
& V_{\text {corrected }}=\text { the corrected, final velocity profile } \\
& V_{\text {offset }}=\text { the constant-velocity offset value } \\
& T=\text { time } \\
& C=\text { cross-axis contamination factor } \\
& V_{\text {long, avg }}=\text { the average longitudinal velocity }
\end{aligned}
$$

The calculated results from the accelerometers were reviewed for "reasonableness". Results from an accelerometer that was inconsistent with the other sensors or with the film and video results were not used to characterize the gross CG motions of the cars or the motions at the coupled ends.

\section{VEHICLE MOTIONS AND FORCES}

The longitudinal, lateral and vertical displacements of the center of gravity of the vehicles were calculated from the double-integrated accelerometer data. Pitch, yaw and roll angular displacements about the CG axes were also calculated.

The longitudinal displacements of the vehicles were calculated by averaging the results from each of the reliable sensors for each vehicle. Figure A-5 shows the longitudinal displacement time-histories of the vehicles based. The impact of the lead car with the barrier occurs at time $=0.0$ seconds. 


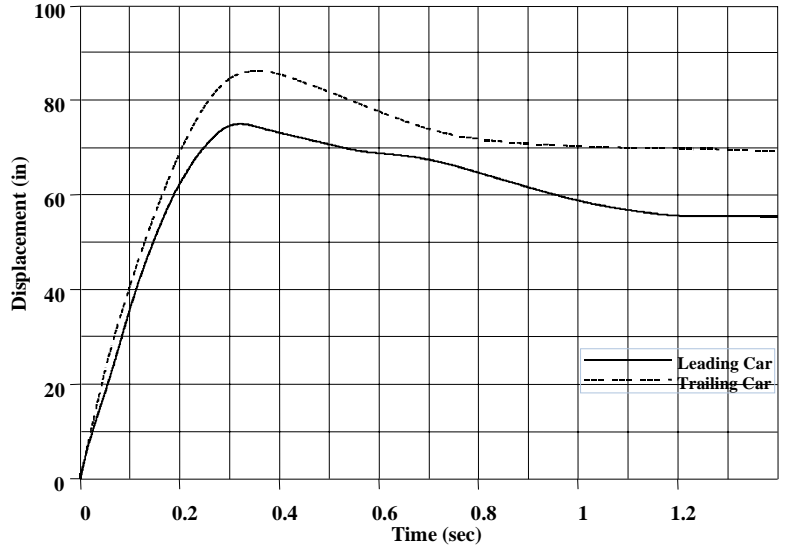

Figure A-5: Longitudinal Displacement of the Lead and Trailing Car Centers of Gravity

The peak longitudinal displacement of the lead car was approximately 75 inches. Due to some elastic behavior at the crush end, the measured crush of the lead car was about 72 inches.[6] The lead car rebounded from the barrier, causing reduced displacements (Figure A-5) after about 0.30 seconds. The trailing car maximum displacement was approximately 86 inches. The larger displacement experienced by the trailing car is due to the additional travel allowed by the draft gear compression, as well as the lateral buckling and lifting motion of the lead end of the trailing car, bringing the coupled ends together briefly. No permanent crush of the trailing vehicle was identified.

The time-history of the longitudinal gap between the ends of the vehicles is calculated by subtracting the longitudinal displacements of the two vehicles. Figure A-6 is a plot of the time-history of the distance, or gap, between the vehicle ends. Note that a negative gap indicates that the vehicles' end beams overlapped. The plot indicates that the gap between the vehicles closed approximately 250 milliseconds after the impact.

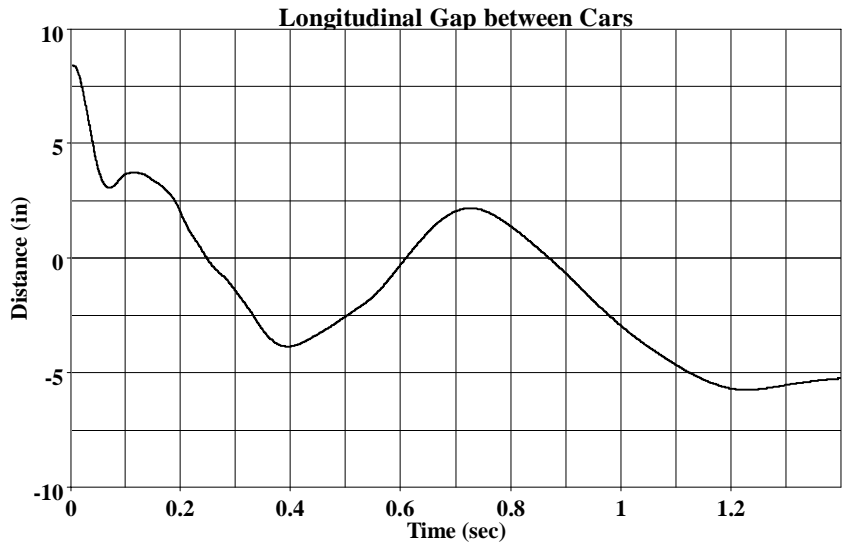

Figure A-6: Time-History of the Longitudinal Distance between the Coupled Ends Cars

Figure A-7 is a plot that shows accelerations at the buffer beams of the car ends between 200 and 400 milliseconds. Also plotted on the graph in Figure A-7 is the gap between the end beams. At about 275 milliseconds we see acceleration spikes in opposite directions indicating that contact may have occurred. Using the masses of the vehicles, the contact force on the lead car is approximately $250,000 \mathrm{lbf}$ and $780,000 \mathrm{lbf}$ on the trailing car.

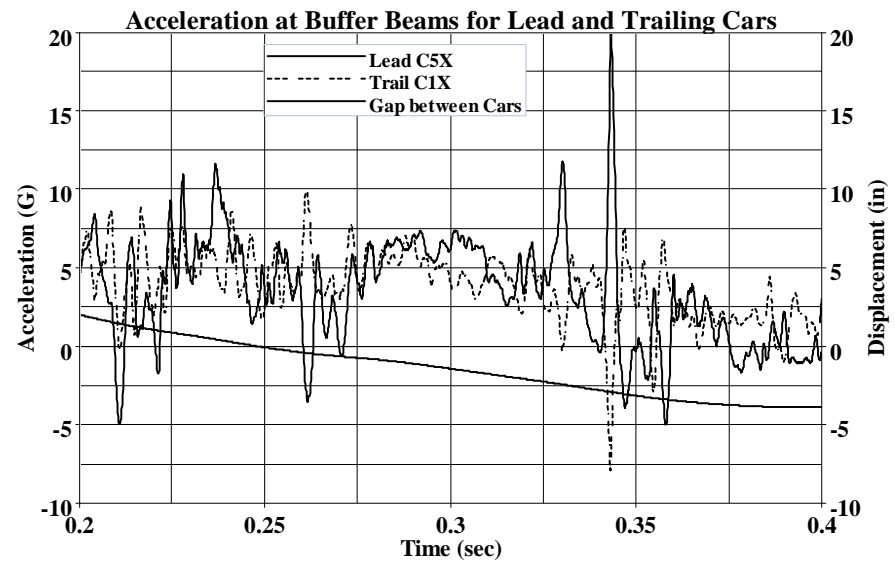

Figure A-7: Vehicle Longitudinal Acceleration and Coupled Car Gap Time-Histories

Lateral and vertical displacements at the coupled ends were calculated using the geometry, displacements, and rotations of the vehicles. The method for determining the displacements was similar for lateral and vertical displacements. The displacement of a point on the centerline at the coupled end of each vehicle is calculated by taking the sum of the lateral displacement of the center of gravity of the vehicle and the geometric displacement of the end due to the rotation of the vehicle. Figure A-8 is a plot of the time-history of the lateral distance of the trailing car end relative to the lead car end. During the formation of lateral buckling, the center points of the ends of the cars moved apart approximately 24 inches before coming to rest 13 inches apart. Figure A-9 shows the relative vertical distance time-history between the center of the vehicle ends. A positive value means the end beam of the trailing car is at a higher elevation than the end beam of the lead car. Note that the trailing car end beam was approximately eight inches above the lead car's end beam near the time it is believed that contact occurred ( 0.3 seconds). Figure A-10 shows post-test evidence that the two vehicles made contact during the test. The figure shows contact marks on the lead car made by the trailing car. The trailing car end may have contacted in a glancing blow as its lead end rose above the lead car's B-end.

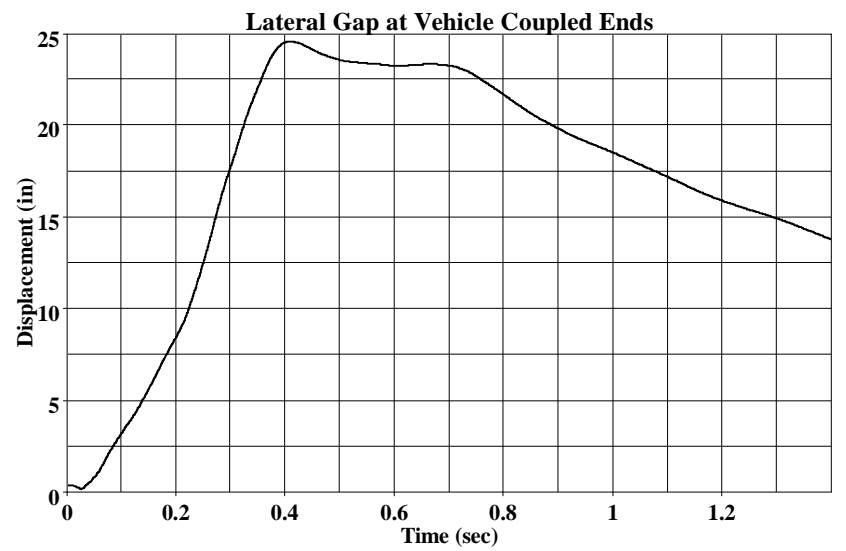

Figure A-8: Relative Lateral Displacement Time-History at the Coupled End 


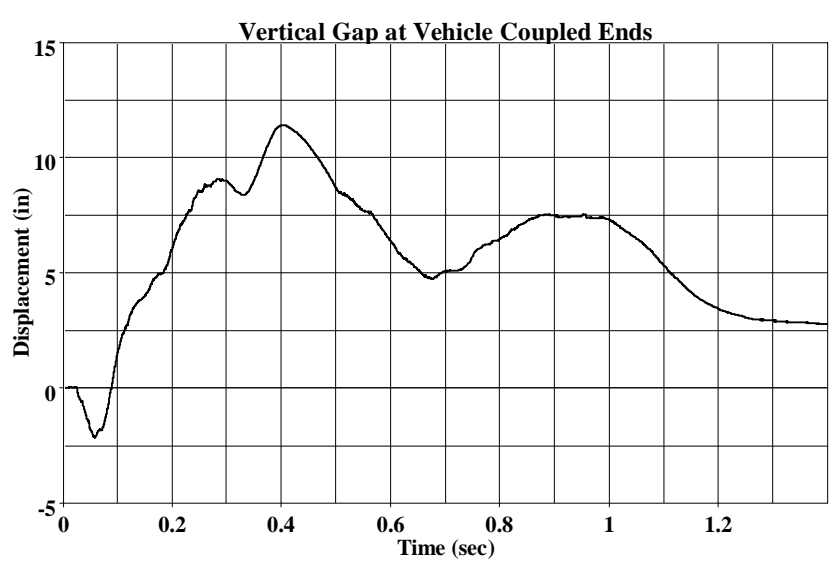

Figure A-9: Relative Vertical Displacement Time-History at the Coupled End

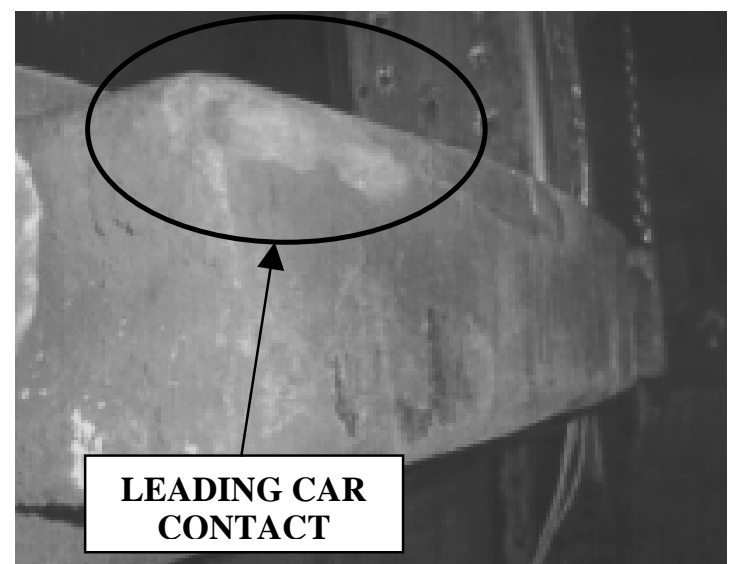

Figure A-10: Contact Marks on the Lead Car Buffer Beam

The post-test investigation indicated that the coupler contacted the upper left corner of the bellmouth of the lead car at the coupled end. Figure A-11 shows indications of the contact that occurred when the trailing car A-end rose above and moved laterally to its left relative to the B-end of the lead car. These contact marks are indications that the upward and forward motions of the trailing car was stopped by the trapping of the coupler below the underframe.

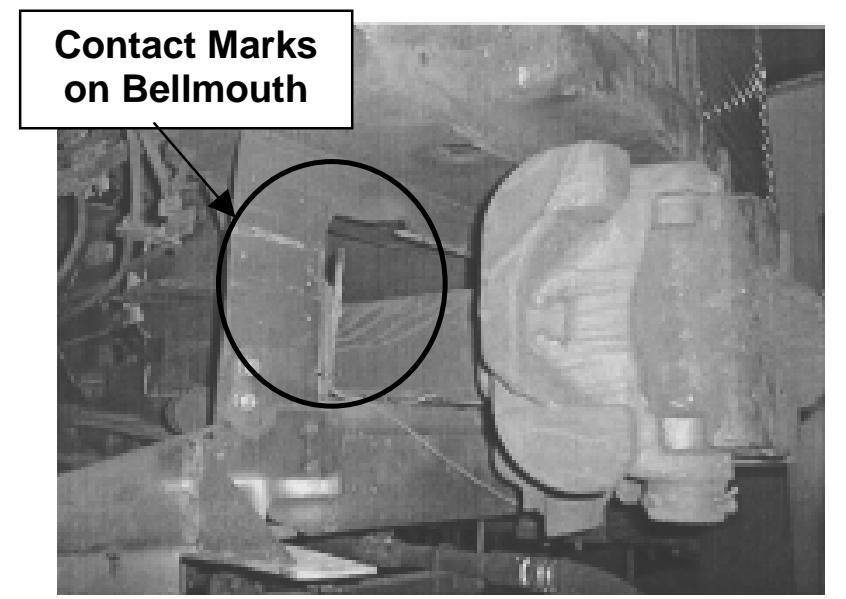

Figure A-11: Contact Marks on the Bellmouth of the Lead Car Following the Inline Test
The magnitude of the forces imposed on the couplers at coupled ends during a collision can aid in understanding the forces that generate derailments and lateral buckling. A strain gage was mounted on the shaft of the coupler to measure the inline load during the test. Figure A-12 shows a time-history plot of the measured inline coupler load. A peak load of approximately $870,000 \mathrm{lbf}$ was measured. This force developed as the load on the lead car peaked. As the force level dropped off on the lead car, the load on the coupler was reduced. The trailing car surged forward and a secondary peak force developed just after 0.2 seconds. As the lateral buckling develops and the coupler pivots, the in-line load reduces. When the vehicles come to rest, the ends of the cars are in a saw-tooth lateral bucking mode and a residual load exists on the coupler due to the compression in the draft gears.

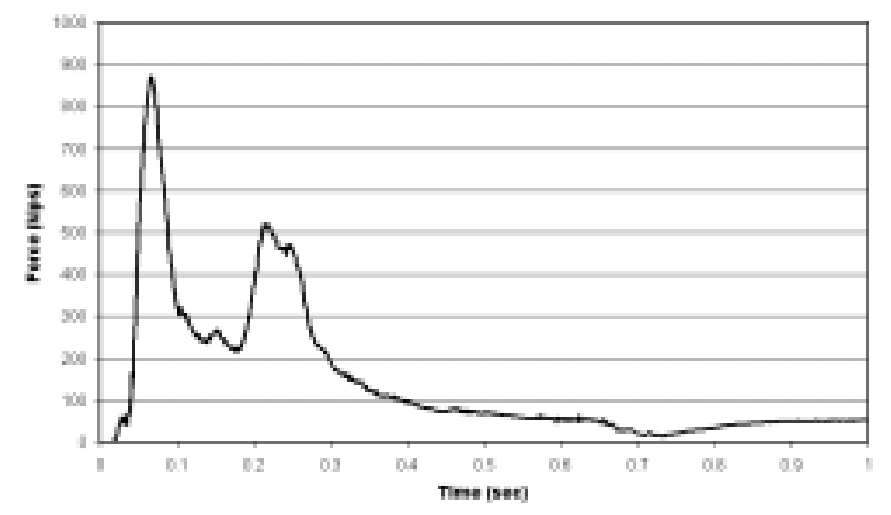

Figure A-12: Longitudinal Coupler Load Time-History 